\title{
Estructura de los encinares de la sierra de Santa Rosa, Guanajuato, México
}

\section{Oak forest structure in the Sierra de Santa Rosa range, Guanajuato, Mexico}

\author{
Juan Martínez-Cruz ${ }^{1 *}$, Oswaldo Téllez Valdés² y Guillermo Ibarra-Manríquez ${ }^{1}$ \\ ${ }^{1}$ Centro de Investigaciones en Ecosistemas, Universidad Nacional Autónoma de México, Antigua carretera a Pátzcuaro 8701. Col. Ex Hacienda de San \\ José de la Huerta, 58190. Morelia, Michoacán. \\ ${ }^{2}$ Facultad de Estudios Superiores Iztacala, Unidad de Biología, Tecnología y Prototipos. Universidad Nacional Autónoma de México. Apartado Postal \\ 314, Av. de los Barrios 1, Los Reyes Iztacala, Tlalnepantla, 54090, Estado de México. \\ *Correspondencia:jmc@oikos.unam.mx
}

\begin{abstract}
Resumen. Se describe la estructura de 4 asociaciones de encinares en el oriente de la sierra de Santa Rosa, Guanajuato, con el método de cuadrantes al punto central (21 transectos), donde se contabilizaron 2320 individuos arbóreos y arbustivos, que incluyen 36 especies, 22 géneros y 15 familias. La asociación Quercus potosina-Q. castanea (QPC) registró la mayor riqueza (29 especies) y el menor número de individuos por ha ${ }^{-1}$ (650). Por el contrario, la asociación de $Q$. laurina- $Q$. rugosa (QLR) presentó sólo 12 especies, pero la mayor densidad de arbustos por ha-1 (5 593). El índice $\alpha$ de Fisher y el análisis de rarefacción ratificaron que la asociación de QPC es la más diversa. La asociación Q. potosina-Q. eduardii (QPE) fue la de mayor equidad. Los índices de Simpson (cualitativo) y de Sørensen (cuantitativo) coincidieron en que las asociaciones $Q$. coccolobifolia-QLR y QLR-QPE presentan la mayor diversidad beta. Debido al marcado deterioro que se observa en estos bosques, la heterogeneidad de las asociaciones de encinares respecto a su composición, atributos de estructura y diversidad, deben considerarse en acciones futuras encaminadas a lograr un manejo más sustentable de los recursos naturales que albergan estos bosques, así como acciones particulares de conservación y restauración.
\end{abstract}

Palabras clave: bosque de Quercus, cuadrantes al punto central, diversidad alfa, diversidad beta, rarefacción.

\begin{abstract}
The structure of 4 oak associations in the western part of the Sierra de Santa Rosa range in Guanajuato state is described. Oak associations were sampled using the point centered quarter method (21 sites). We censused 2320 individuals of trees and shrubs belonging to 36 species, in 22 genera and 15 families. Observed species richness (29) was highest in the Quercus potosina-Q. castanea association (QPC) but tree density was the lowest (650 individuals/ha). Meanwhile, the Q. laurina-Q. rugosa association (QLR) had the least number of observed species (12), but the maximum shrub density (5 593 individual/ ha). The Fisher index for alpha diversity and the rarefaction analysis confirmed the QPC as the most diverse association. On the other hand, the Q. potosina-Q. eduardii association (QPE) had the highest value of equitability among the oak-forest associations analyzed. Based on the Simpson (presence-absent) and Sørensen abundance index values, we find that beta diversity is high across the $Q$. coccolobifolia and QLR associations and also the QLRQPE ones. Due to the high degree of disturbance within these forests, mostly of anthropogenic origin, the heterogeneity in oak associations as well as their structure and diversity ought to be considered in future conservation and restoration management plans.
\end{abstract}

Key words: alfa diversity, beta-diversity, point centered quarter method, Quercus forest, rarefaction.

\section{Introducción}

El estado de Guanajuato es de los más densamente poblados de México, debido a que gran parte de su territorio ha sido escenario de una intensa actividad minera, explotación agrícola, desarrollo industrial y establecimiento de áreas urbanas desde la época de la Colonia (Carranza, 2005). Este prolongado e intenso uso por el ser humano, debido en particular a la agricultura

Recibido: 15 octubre 2007; aceptado: 28 julio 2008 de riego y temporal, ha afectado profundamente la vegetación natural, la cual está severamente modificada, degradada o incluso ha desaparecido en el $49.2 \%$ de sus 30629 km² (Quero, 1977; Pineda, 1978; Rzedowski et al., 1996; Palacio-Prieto et al., 2000). Hace poco más de una década, Guanajuato, Campeche, Colima y Zacatecas, eran consideradas como entidades deficientes en el conocimiento de su flora y vegetación (Dávila y Sosa, 1994). Por fortuna para Guanajuato, la situación ha mejorado en los últimos años con el desarrollo del proyecto florístico de la Región del Bajío y Zonas Adyacentes, que fue iniciado 
en 1991 y que hasta la fecha consta de 154 monografías. Villaseñor (2003) indicó que la flora de Guanajuato estaba compuesta por 143 familias, 722 géneros y 1755 especies, mientras que Carranza (2005) la estimó en 2547 especies, considerando que hasta ahora se ha avanzado alrededor del $50 \%$ en su inventario.

Por otro lado, el panorama respecto al conocimiento de los atributos estructurales de las comunidades vegetales de Guanajuato tiene menor avance que el mencionado para la flora, ya que los trabajos relacionados se limitan principalmente a información de tipo fisonómico, en los que se destacan las especies más características de cada tipo de vegetación (McVaugh y Rzedowski, 1966; Aguilera, 1991; Rubio, 1993; Rzedowski et al., 1995, 1996; Martínez-Cruz y Téllez, 2004; Carranza, 2005). La información general sobre abundancia de especies leñosas, en especial de las arbóreas, puede encontrarse en Quero (1977), Pineda (1978), Rivas (1980) y Rzedowski et al. (1996).

Lo anterior justifica con amplitud el realizar estudios que describan con más detalle la composición, estructura y diversidad de las comunidades vegetales de Guanajuato, en especial de los bosques de encino ( $9.3 \%$ de su territorio) y de pino-encino $(2.9 \%)$, que en conjunto representan la mayor proporción de vegetación natural que hay en la actualidad en esa entidad (Palacio-Prieto et al., 2000). Cabe destacar que en México, estos ecosistemas son altamente diversos, con un 34\% de la flora estimada para el país (Rzedowski, 1991). Para el caso particular de la sierra de Santa Rosa, la vegetación fisonómica y territorialmente más conspicua es el bosque de encino, con una cobertura del 60 al $80 \%$ (Quero, 1977; Pineda, 1978). Un antecedente florístico importante para el área es el de Martínez-Cruz y Téllez (2004), quienes aportan una lista de 496 especies, incluidas en 273 géneros y 93 familias, en tanto que la estructura de los encinares es prácticamente desconocida. Con base en lo anterior, los objetivos del presente trabajo son: i) describir la estructura y diversidad de las comunidades vegetales de los bosques de encino de la porción oriental de la sierra de Santa Rosa, en el estado de Guanajuato y ii) comparar estos atributos con comunidades similares ubicadas dentro y fuera de México.

\section{Materiales y métodos}

Área de estudio. La sierra de Santa Rosa se localiza en los alrededores de la ciudad de Guanajuato, entre los $20^{\circ} 45^{\prime}$ $21^{\circ} 25^{\prime} \mathrm{N}$ y $\operatorname{los} 100^{\circ} 53^{\prime}-101^{\circ} 25^{\prime} \mathrm{O}$ (Fig. 1), formando parte de los municipios de Dolores Hidalgo, Guanajuato y San Felipe (INEGI, 1991). Esta sierra se encuentra inmersa dentro de la subprovincia de sierras y llanuras del

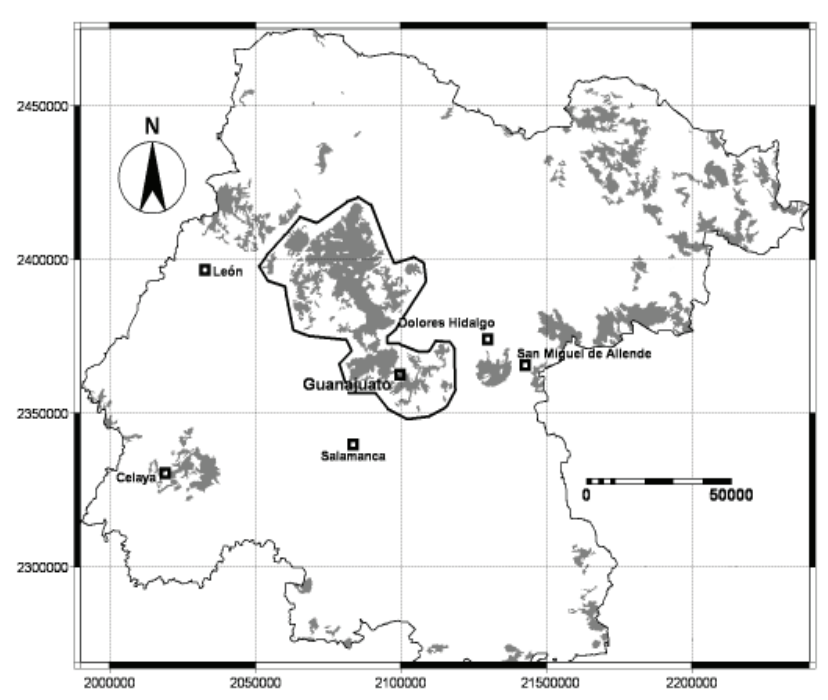

Figura 1. Localización de la sierra de Santa Rosa, cerca de la ciudad de Guanajuato (polígono en línea gruesa). En tonos grises se muestra la distribución de los bosques de encino en el estado.

norte de Guanajuato, perteneciente a la región fisiográfica de la mesa central (SPP, 1981; Cuanalo de la Cerda et al., 1989), con un gradiente altitudinal que va de 1800 a 2 $750 \mathrm{~m}$ y montañas fuertemente disectadas. La geología de esta sierra corresponde al periodo Cretácico, con rocas ígneas, principalmente andesitas, basaltos, ignimbritas, riolitas y tobas, así como rocas sedimentarias formadas por materiales detríticos (Cuanalo de la Cerda et al., 1989; Ferrusquía-Villafranca, 1993). La zona de estudio presenta un clima $\mathrm{C}(\mathrm{w})(\mathrm{w})$, templado subhúmedo con lluvias en verano, precipitación invernal menor del $5 \%$ respecto a la precipitación promedio anual, que es de $800 \mathrm{~mm}$, y un periodo de sequía de 7 meses (noviembre a mayo), en el cual diciembre es el más seco (>10 mm), en tanto que agosto se registra como el más lluvioso (170-180 mm) (SPP, 1981; García, 1981). En el aspecto hidrológico, esta sierra se encuentra inmersa en la cuenca LermaSalamanca y el único cuerpo de agua en la zona es la presa La Esperanza, que surte de agua a la capital del estado (SPP, 1981). El tipo de vegetación descrito en la literatura para el sitio de estudio es el bosque de encino, donde destaca florísticamente el género Quercus (14 especies), con especies leñosas acompañantes pertenecientes a los géneros Ageratina, Arbutus, Comarostaphylis y Solanum (Pineda, 1978; Rzedowski, 1978; Martínez-Cruz y Téllez, 2004).

Muestreo. La obtención de los parámetros estructurales se realizó por el método de punto cuadrante (MuellerDombois y Ellenberg, 1974). Los sitios de muestreo se seleccionaron por medio de recorridos a lo largo del área 
de estudio, tomando como referencia diferentes variantes fisonómicas de los encinares, en particular la altura y la composición de las especies arbóreas y arbustivas más abundantes, así como su extensión territorial. En cada sitio reconocido con estos criterios, se realizaron transectos de 100 o $200 \mathrm{~m}$, donde se ubicó el mismo número de puntos de muestreo para árboles y arbustos, separados entre sí cada 10 y 5 m, respectivamente. En cada punto se trazó una línea imaginaria, perpendicular al transecto, para obtener 4 cuadrantes y dentro de cada uno de éstos se consideró el árbol o arbusto más cercano al punto de intersección de las líneas. Los individuos fueron determinados en el campo y se midió su distancia respecto al punto central. La dominancia (biomasa) en los árboles fue estimada por medio del diámetro del tronco, medido a una altura de 1.3 $\mathrm{m}$ respecto al suelo (d.a.p.), mientras que para los arbustos se evaluó por medio de la cobertura de la copa, midiendo primero su eje más largo, así como el eje perpendicular mayor a éste. Se consideraron como árboles las plantas leñosas cuya primera ramificación se localizó arriba de $0.5 \mathrm{~m}$, mientras que los arbustos incluyen los individuos leñosos que se ramificaron a una altura menor.

Con la información agrupada de todos los transectos para cada asociación vegetal y siguiendo los criterios propuestos por Mueller-Dombois y Ellenberg (1974), se estimaron los siguientes valores de estructura: i) la separación espacial entre individuos (D), que es calculada como $\sum \mathrm{d}_{i \ldots \mathrm{n}} / \mathrm{N}$, donde $\mathrm{d}_{\mathrm{i}}$ es la distancia de la $i$ especie al centro del transecto y $\mathrm{N}$ es el número total de individuos); ii) la densidad por hectárea, que se obtiene del cociente entre el área de la que se desea estimar la densidad de árboles (en este caso $10000 \mathrm{~m}^{2}$ ) y D $\mathrm{D}^{2}$; iii) área basal de la especie, que es la sumatoria del área basal del total de sus individuos censados en los transectos, y iv) el índice de importancia por especie, que se obtiene de la sumatoria de su densidad, dominancia y frecuencia relativas. Por último, los perfiles de las asociaciones vegetales se realizaron modificando la propuesta de Danserau (1957), en la que se representa únicamente la forma de crecimiento, la altura y fenología (caducifolios o perennifolios durante la época seca) de las especies arbóreas y arbustivas.

Diversidad. El análisis de esta variable entre las asociaciones reconocidas para este estudio se obtuvo usando primeramente el número de especies local $(\mathrm{S})$. Adicionalmente, se calculó el índice alfa $(\alpha)$ de la serie logarítmica $(\alpha=\mathrm{N}(1-\mathrm{x}) / \mathrm{x})$, donde $\mathrm{N}$ es el número total de individuos registrados en el sitio de muestreo y la variable $\mathrm{x}$ es obtenida a partir de la siguiente fórmula:

$\mathrm{S} / \mathrm{N}=[(1-\mathrm{x} / \mathrm{x}][-\ln (1-\mathrm{x})]$. Magurran (2004) considera este algoritmo como adecuado para estimaciones de diversidad, ya que lo afecta poco el tamaño de la muestra y cuando $\mathrm{N}>1000$, sus valores son completamente independientes de esta variable. Para calcular el valor de este índice se utilizó el programa BiodiversityPro® (McAleece, 1997). Se obtuvo el índice de ShannonWiener, cuya fórmula es (Moreno, 2001; Magurran, 2004): $\mathrm{H}^{\prime}=-\sum \mathrm{p}_{i} \ln \mathrm{p}_{i}$, donde $\mathrm{p}_{i}=$ proporción de individuos de la $i$ th especie y ln es el logaritmo natural de $\mathrm{p}_{\mathrm{i}}$. El índice presupone que los individuos son elegidos aleatoriamente de una comunidad infinita y que todas las especies están incluidas en el análisis, lo que normalmente no acontece en los trabajos sobre estructura de comunidades. Debido a lo anterior y a que sus valores están influidos por el tamaño de la muestra, Magurran (2004) desestima su uso y recomienda en su lugar otros índices. Sin embargo, se decidió calcularlo con el objeto de hacer comparaciones con trabajos previos realizados en encinares y para obtener valores de equidad de las comunidad (véase el siguiente párrafo).

Para obtener una estimación de la equidad de las asociaciones, primeramente se calculó el índice de Simpson $\left(\mathrm{D}=\sum_{\mathrm{i}}\left[\mathrm{n}_{\mathrm{i}}\left(\mathrm{n}_{\mathrm{i}}-1\right) / \mathrm{N}(\mathrm{N}-1)\right]\right)$, donde $\mathrm{n}_{\mathrm{i}}$ es el número de individuos de la especie ${ }_{i}$ y $\mathrm{N}$ es el total de individuos censados en el sitio. El índice D sopesa sus valores por las especies con mayor densidad en la muestra e indica la probabilidad de que 2 individuos tomados al azar de una comunidad finita pertenezcan a la misma especie (Moreno, 2001; Magurran, 2004). La equidad es una medida de uniformidad en la densidad de las especies de una comunidad. Esta variable puede calcularse con base en el valor del inverso de $\mathrm{D}$ y el número de especies del sitio $\left[\mathrm{E}_{1 / \mathrm{D}}=(1 / \mathrm{D}) / \mathrm{S}\right]$, lo que puede indicar un gradiente de nula (0) a máxima (1) equidad. También puede obtenerse a partir del índice de Shannon-Wiener, señalan Moreno (2001) y Magurran (2004), en el supuesto de que la diversidad máxima $\left(\mathrm{H}_{\max }\right)$ debería presentarse cuando las especies de una comunidad tienen una abundancia igual $\left(\mathrm{H}^{\prime}=\mathrm{H}_{\max }=\ln \mathrm{S}\right)$; en consecuencia, la equidad puede obtenerse al dividir la diversidad observada ( $\left.\mathrm{H}^{\prime}\right)$ entre la $\mathrm{H}_{\max }\left(\mathrm{H}^{\prime} / \ln \mathrm{S}\right)$. Se realizó una prueba de $t$ para determinar si existían diferencias estadísticamente significativas entre los valores de H' entre las distintas asociaciones definidas en el presente estudio (Magurran, 2004).

La riqueza de especies de una comunidad depende, entre otros factores, del área censada o el número de los individuos registrados, como es el caso de las asociaciones reconocidas para la sierra de Santa Rosa. Para que las comparaciones no estén influidas por estas variables, Magurran (2004) sugiere hacer un análisis de rarefacción, en el cual los valores de S son comparados tomando como punto de referencia el del sitio que tenga el menor número de individuos. Este ejercicio se realizó con el programa EstimateS (Colwell, 2005) y además se obtuvieron curvas de acumulación de especies con una varianza asociada a un 
nivel de confianza del $95 \%$, usando las formulas de Colwell et al. (2004), a partir de remuestreos (50 iteraciones) de las especies de cada asociación. Estas curvas, entonces, se comparan tomando también como base la asociación con la menor abundancia y en caso de que los valores de confianza no se traslapen, se considera que difieren en la variable $\mathrm{S}$.

Por otro lado, la diversidad beta ha sido definida como el reemplazamiento o cambio de especies que se presenta entre 2 sitios en una escala espacial o temporal determinada (Moreno, 2001; Halffter y Moreno, 2005), aunque es importante destacar que existen diferentes definiciones para este complejo concepto (Koleff, 2005). Considerando propuestas recientes en la literatura (Koleff et al., 2003; Koleff, 2005), la diversidad beta fue calculada con un índice de presencia-ausencia de especies (Simpson, $\mathrm{B}_{\mathrm{SIM}}$ ) que se obtiene con la fórmula:

$\mathrm{B}_{\mathrm{SIM}}=1-\mathrm{A} / \min (\mathrm{B}, \mathrm{C})+\mathrm{A}$

donde A corresponde a la cantidad de especies compartidas entre 2 muestras, B y C representan el número de especies registradas únicamente en la muestra 1 y 2 , respectivamente, en tanto que min es el valor mínimo de especies restringidas en las muestras que se comparan (B o C). Este índice presenta sus valores máximos (una alta diversidad beta) cuando el porcentaje de especies que se comparten es bajo y los porcentajes de ganancia-perdida entre las áreas que se comparan son similares (Koleff et al., 2003; Koleff, 2005).

Magurran (2004) sugiere también obtener la diversidad beta con un índice cuantitativo, es decir, que considere el número de individuos por especie entre 2 sitios de muestreo, para lo cual recomienda el Sørensen cualitativo $\left(\mathrm{S}_{\mathrm{SOR}}\right)$, también conocido como el índice de Bray-Curtis; este índice se calculó con el software EstimateS (Colwell, 2005), con base en la siguiente fórmula (Moreno, 2001):

$$
\mathrm{S}_{\mathrm{SOR}}=2 p N / a N+b N
$$

donde $a N$ y $b N$ es el número total de individuos en el sitio A y $\mathrm{B}$, respectivamente y $p N$ es la sumatoria de la abundancia más baja registrada de las especies que se comparten entre los sitios comparados. Cuando este índice tiende hacia 1 indica que las muestras que se comparan tienen una alta similitud y en consecuencia una baja diversidad beta (Magurran, 2004).

\section{Resultados}

Los recorridos a lo largo de la sierra de Santa Rosa permitieron distinguir 4 asociaciones (Apéndice 1): i) Quercus coccolobifolia, ii) $Q$. laurina-Q. rugosa, iii) $Q$. potosina-Q. castanea y iv) $Q$. potosina- $Q$. eduardii. Dentro de estas asociaciones y de acuerdo a su extensión se distribuyeron 21 transectos y se midieron 2320 individuos (1 160 árboles y 1160 arbustos), que incluyen 36 especies, 22 géneros y 15 familias (Apéndice 1).

La asociación de $Q$. coccolobifolia se desarrolló dentro de un gradiente altitudinal de 2000 a $2600 \mathrm{~m}$, en sitios con elevada exposición solar, sobre afloramientos rocosos, con una distribución muy restringida, por lo que sólo fue posible realizar en ésta 2 transectos de $200 \mathrm{~m}$, contabilizando 13 especies (320 individuos, 160 árboles y 160 arbustos). La densidad de árboles estimada fue de 1246 ind. ha ${ }^{-1}$, separados en promedio cada $2.8 \mathrm{~m}$ y con un área basal promedio por individuo de $0.7 \mathrm{~m}^{2}$. Quercus coccolobifolia es la especie más abundante entre las 6 especies arbóreas con una densidad estimada de 1067 ind. ha ${ }^{-1}$, un área basal de $694.1 \mathrm{~m}^{2}$ ha $^{-1}$ y un índice de importancia de $77.3 \%$ (Apéndice 1). Pinus cembroides (Pinaceae) fue la especie con el valor máximo de d.a.p. $(23.9 \mathrm{~cm})$. El componente arbustivo incluye 10 especies, destacando por su densidad y dominancia Arcostaphylos pungens (Ericaceae) y Q. coccolobifolia (Cuadro 1). La densidad estimada para los arbustos fue de 2544 ind. ha-1, una cobertura promedio de $0.9 \mathrm{~m}^{2}$ y una distancia promedio entre individuos de $1.9 \mathrm{~m}$. La altura del componente arbóreo y arbustivo fue homogénea y osciló entre 3-10 m y 0.5-2 m, respectivamente; 5 especies fueron caducifolias, 3 de ellas pertenecientes al género Quercus (Fig. 2a).

La segunda asociación ( $Q$. laurina-Q. rugosa) también se distribuye de manera muy limitada, principalmente en las partes más altas y húmedas del área de estudio (2 600$2750 \mathrm{~m}$ de altitud), en pendientes mayores de $30^{\circ}$, por lo que sólo se realizó un transecto de 100 m y otro de 200 m (320 individuos, 160 árboles y 160 arbustos). A pesar de que en esta asociación se registró el menor número de especies (12) en el área de estudio, presentó el segundo valor estimado más alto de árboles por hectárea (1 133), con una separación promedio de $2.9 \mathrm{~m}$ y un área basal promedio de $3.0 \mathrm{~m}^{2}$. Las especies Quercus laurina y $Q$. rugosa, las únicas del género detectadas en esta asociación, presentaron densidades similares por hectárea, pero su área basal fue bastante contrastante, lo que se refleja en sus índices de importancia (Apéndice 1); la especie Q. laurina registró al individuo con mayor diámetro $(59.2 \mathrm{~cm})$. Respecto a los arbustos (10 especies), cabe señalar que en esta asociación se obtuvo la mayor densidad de individuos por hectárea (5 593) y obviamente la separación más baja entre éstos $(1.3 \mathrm{~m})$, con una elevada presencia de plántulas e individuos jóvenes de $Q$. laurina; 3 de las 4 especies con mayor índice de importancia en este estrato pertenecen 

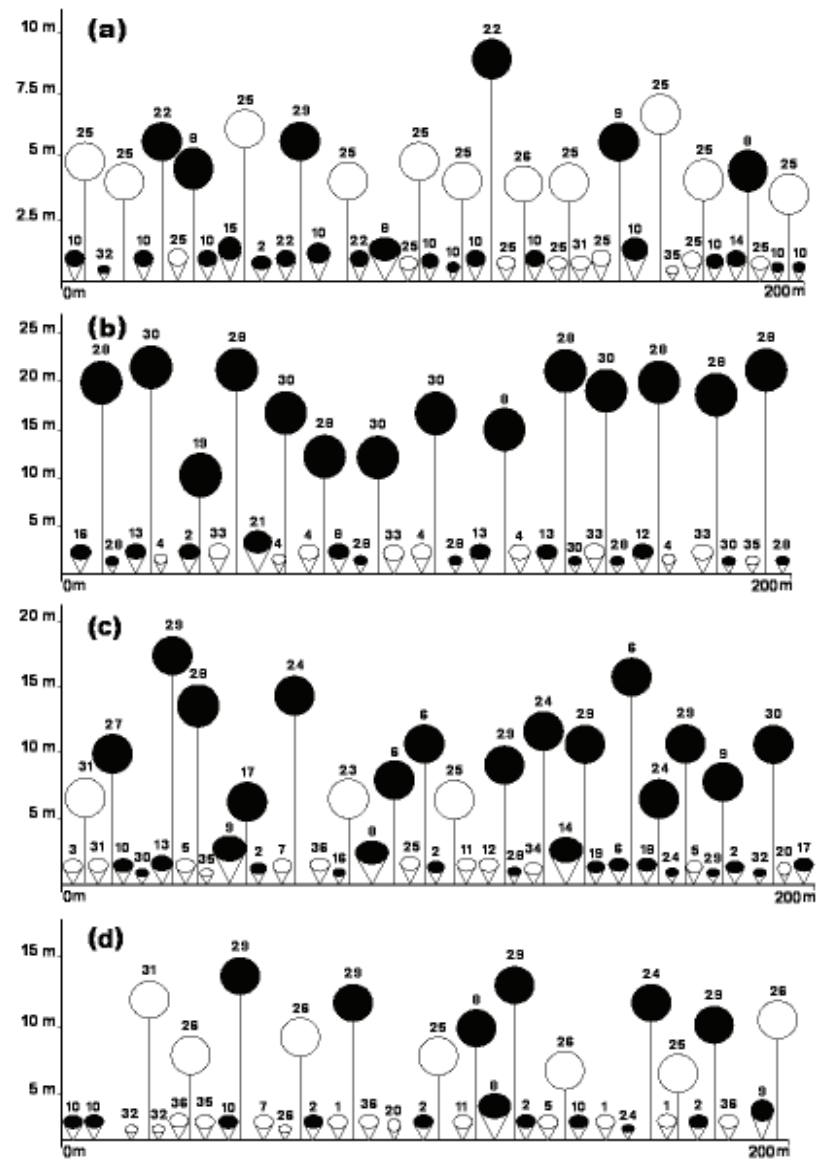

Figura 2. Danserogramas de las asociaciones de encino encontradas en el área de estudio: a) Quercus coccolobifolia, b) Quercus laurina-Q. rugosa, c) Quercus potosina- $Q$. castanea y d) Quercus potosina-Q. eduardii. Simbología: árbol 9 , arbusto $\boldsymbol{\theta}$, perennifolio $\bullet$, y caducifolio $\bigcirc$. Especies: 1, Ageratina calaminthaefolia; 2, A. glabrata; 3, A. petiolaris; 4, Ageratina sp. 1; 5, Ageratina sp. 2; 6, Alnus acuminata; 7, Amelanchier denticulata; 8, Arbutus glandulosa; 9, A. xalapensis; 10, Arctostaphylos pungens; 11, Baccharis heterophylla; 12, Buddleja cordata; 13, Cestrum anagyris; 14 , Comarostaphylis glaucescens; 15, C. polifolia; 16, Cornus excelsa; 17, Crataegus mexicana; 18, Dodonaea viscosa; 19, Garrya laurifolia; 20, Havardia elachystophylla; 21, Ilex dugesii; 22, Pinus cembroides; 23, Prunus serotina; 24, Quercus castanea; 25, Q. coccolobifolia; 26, Q. eduardii; 27, Q. laeta; 28, Q. laurina; 29, Q. potosina; 30, Q. rugosa; 31, Q. sideroxyla; 32, Rhamnus microphylla; 33, Roldana angulifolia; 34, Salvia mexicana; 35, Stevia lucida; 36, S. salicifolia.

a Asteraceae (Apéndice 1). La cobertura promedio por individuo para esta forma de crecimiento es la de menor valor entre las asociaciones descritas en este trabajo ( 0.4 $\mathrm{m}^{2}$ ). El estrato arbóreo de esta asociación es el más alto registrado para la sierra de Santa Rosa, alcanzando en varios de sus individuos hasta $25 \mathrm{~m}$, mientras que los arbustos no sobrepasan los $4 \mathrm{~m}$; únicamente la especie arbustiva Roldana angulifolia (Asteraceae) fue registrada como caducifolia (Fig. 2b).

Contrariamente a las 2 asociaciones vegetales anteriores, la de $Q$. potosina- $Q$. castanea es la de mayor distribución en el área estudiada, dentro de un intervalo altitudinal de 2300 a $2600 \mathrm{~m}$ snm, sobre pendientes de hasta $30^{\circ}$. En esta asociación se realizaron 8 transectos de $100 \mathrm{~m}$ y 4 de $200 \mathrm{~m}$, en los que se registraron 29 especies y un total de 1280 individuos (640 árboles y 640 arbustos). La densidad estimada para la forma de crecimiento arbórea fue de 650 ind. ha ${ }^{-1}$, con una separación espacial de 3.9 m y un área basal promedio de $1.9 \mathrm{~m}^{2}$; los individuos de $Q$. potosina fueron los que alcanzaron el mayor valor de d.a.p. (30 cm). Las especies $Q$. potosina y $Q$. castanea sobrepasan con claridad por número de individuos y área basal al resto de las especies con las que cohabitan en el estrato arbóreo, incluyendo 5 especies más de encinos (Apéndice 1). Algunas especies de árboles presentes en esta asociación vegetal y que debido a su poca densidad no fueron detectadas por el muestreo fueron Ceanothus coeruleus (Rhamnaceae), Garrya laurifolia (Garryaceae) e Ilex dugesii (Aquifoliaceae), las cuales se observaron únicamente en arroyos pequeños. Por otro lado, aunque los arbustos de esta asociación no son los más abundantes en toda el área de estudio (5089 ind. ha ${ }^{-1}$ ), sí son los de mayor cobertura promedio $\left(1.1 \mathrm{~m}^{2}\right)$, con una separación espacial de $1.4 \mathrm{~m}$. Entre los arbustos, 3 de las 4 especies con mayor índice de importancia pertenecen a Asteraceae (Apéndice 1), destacando al respecto Ageratina glabrata. Los individuos arbóreos más altos fueron de Q. potosina y Alnus acuminata (Betulaceae), con un estrato arbustivo que no sobrepasa los $5 \mathrm{~m}$, particularmente conformado por 2 especies del género Arbutus (Ericaceae); 7 especies fueron observadas con un comportamiento caducifolio, pertenecientes a Asteraceae y Fagaceae (Fig. 2c).

La última asociación registrada estuvo dominada por $Q$. potosina- $Q$. eduardii, y se encontró en pequeñas franjas entre los $2400-2500 \mathrm{~m}$, sobre pendientes de más de $30^{\circ}$ o en pequeñas mesetas. La caracterización de los fragmentos se realizó mediante el trazo de 2 transectos de $100 \mathrm{~m}$ y uno de $200 \mathrm{~m}$, con un registro de 17 especies (320 individuos, 160 árboles y 160 arbustos). Las estimaciones estructurales para esta asociación indican una densidad arbórea de 656 ind. ha ${ }^{-1}$, con una separación de $3.9 \mathrm{~m} \mathrm{y}$ un área basal promedio de $1.9 \mathrm{~m}^{2}$; la especie $Q$. castanea registró el valor máximo registrado para el d.a.p. (38.1 $\mathrm{cm})$. Entre las 6 especies de árboles cuantificadas, 5 pertenecen al género Quercus, entre las que es obvio que sobresalgan $Q$. potosina y $Q$. eduardii, con 47.9 y $28.3 \%$ de índice de importancia, respectivamente (Apéndice 1). Entre las 14 especies que componen el estrato arbustivo, Arctostaphylos pungens y Ageratina glabrata presentaron 
valores similares de índice de importancia (25.6 y 24.3, respectivamente), con 1435 ind. ha ${ }^{-1}$ (Apéndice 1); la cobertura promedio de los arbusto fue de $0.9 \mathrm{~m}^{2}$, con una separación entre éstos de $1.7 \mathrm{~m}$ y una densidad de 3425 ind. ha ${ }^{-1}$. El estrato arbóreo presenta alturas cercanas a los $15 \mathrm{~m}$, donde $Q$. potosina es la especie que despliega los individuos de mayores dimensiones, en tanto que los arbustos raramente sobrepasan los $5 \mathrm{~m}$ (Arbutus glandulosa, Ericaceae); un total de 8 especies mostraron una fenología caducifolia, la mayoría pertenecientes a Asteraceae y Fagaceae (Fig. 2d).

Diversidad. El índice $\alpha$ de Fisher señala que la asociación de Q. potosina-Q. castanea es la más diversa, mientras que $Q$. laurina-Q. rugosa fue la de menor valor, resultados que son consistentes con el número de especies local (Cuadro 1). Por el contrario, en este cuadro se observa que para el índice de Shannon-Wiener, la asociación más importante es la de $Q$. potosina- $Q$. eduardii. De acuerdo con los resultados de la prueba de $t$ (Cuadro 2), las asociaciones reconocidas para el sitio de estudio difieren en sus valores de H', excepto entre la dupla formada por $Q$. potosina- $Q$. eduardii y $Q$. potosina-Q. castanea. Respecto a los índices para calcular la equidad (E y 1/D), la mayor relevancia es para la asociación Q. potosina-Q. eduardii (Cuadro 1).

El análisis de rarefacción resultó más afín a los resultados indicados por el índice $\alpha$ de Fisher, donde la comunidad de $Q$. potosina- $Q$. castanea es la más diversa (Fig. 3). El análisis de las curvas de acumulación de especies aleatorias mostró que varias asociaciones no diferían en la $\mathrm{S}$ estimada (no se muestran las curvas) y que únicamente $Q$. laurina- $Q$. rugosa es significativamente distinta de las asociaciones conformadas por $Q$. potosina- $Q$. castanea (Fig. 4a) y por $Q$. potosina-Q. eduardii (Fig. 4b).

Cuadro 1. Riqueza y diversidad de las asociaciones de encino reconocidas en la sierra de Santa Rosa, Guanajuato

\begin{tabular}{lccccc}
\hline Asociación & $S$ & $\begin{array}{c}\alpha d e \\
\text { Fisher }\end{array}$ & $H^{\prime}$ & $E$ & $E_{1 / D}$ \\
\hline $\begin{array}{l}\text { Quercus } \\
\text { coccolobifolia }\end{array}$ & 13 & 2.725 & 0.567 & 0.22 & 0.19 \\
$\begin{array}{l}\text { Q. laurina- } \\
\text { Q. rugosa }\end{array}$ & 12 & 2.463 & 0.732 & 0.29 & 0.32 \\
$\begin{array}{l}\text { Q. potosina- } \\
\text { Q. castanea }\end{array}$ & $\mathbf{2 9}$ & $\mathbf{5 . 2 8 2}$ & 1.022 & 0.30 & 0.25 \\
$\begin{array}{l}\text { Q. potosina- } \\
\text { Q. eduardii }\end{array}$ & 17 & 3.834 & $\mathbf{1 . 0 3 4}$ & $\mathbf{0 . 3 6}$ & $\mathbf{0 . 4 9}$ \\
\hline
\end{tabular}

Abreviaturas: $S$, número de especies; $H^{\prime}$, índice de ShannonWiener; $E$, equidad con base en $\mathrm{H}^{\prime} ; E_{I / D}$, equidad basada en el índice de Simpson. Los valores más altos detectados en cada asociación se resaltan en negritas.
Respecto a la diversidad beta, el índice de Simpson (presencia-ausencia) señala que la asociación $Q$. laurina- $Q$. rugosa presentó con otras 2 asociaciones ( $Q$. coccolobifolia y $Q$. potosina- $Q$. eduardii) valores igualmente importantes respecto a esta variable, un resultado congruente con lo que indica el índice cuantitativo de Sørensen (Cuadro 3). Otra manera de visualizar la heterogénea composición de estas asociaciones es que únicamente Ageratina glabrata, Arbutus glandulosa y Stevia lucida (Asteraceae) fueron registradas en todas ellas, mientras que 13 especies restringieron su distribución a sólo una asociación (Apéndice 1).

\section{Discusión}

Las especies que conforman las 4 asociaciones mencionadas en el texto se han registrado entre los componentes más conspicuos de los encinares en Guanajuato (McVaugh y Rzedowski, 1966; Quero, 1977; Pineda, 1978; Aguilera, 1991; Rzedowski et al., 1996). En particular, Quero (1977) señaló que los encinares con mayor área de distribución del NO de Guanajuato se encuentran dominados por $Q$. potosina y $\mathrm{Q}$. eduardii, mientras que aquellos donde son más importantes las especies de $Q$. laurina y $Q$. rugosa desplegaban una distribución más restringida, lo que coincide con los resultados presentados en el presente trabajo.

La fisonomía de los encinares en esta sierra no se aleja marcadamente del patrón que se ha encontrado en estudios similares (Rzedowski, 1978; Rivas, 1980; Rubio, 1993; Kappelle, 1996; Márquez et al., 1999; Enríquez, 2003), ya que presentan una disposición vertical con sus componentes

Cuadro 2. Valores de la prueba de $t$ para los resultados obtenidos con el índice de Shannon en cada asociación de este estudio*

\begin{tabular}{|c|c|c|c|}
\hline Asociación & $Q C O$ & $Q L R$ & $Q P C$ \\
\hline $\begin{array}{l}\text { Quercus } \\
\text { coccolobifolia } \\
\text { (QCO) }\end{array}$ & -- & & \\
\hline $\begin{array}{l}\text { Quercus laurina- } \\
\text { Q. rugosa (QLR) }\end{array}$ & $\begin{array}{c}4.237> \\
\mathrm{t}_{(616)}=3.306\end{array}$ & -- & \\
\hline $\begin{array}{l}\text { Quercus potosina- } \\
\text { Q. castanea } \\
\text { (QPC) }\end{array}$ & $\begin{array}{c}13.809> \\
\mathrm{t}_{(449)}=3.312\end{array}$ & $\begin{array}{c}10.330> \\
\mathrm{t}_{(516)}=3.310\end{array}$ & -- \\
\hline $\begin{array}{l}\text { Quercus potosina- } \\
\text { Q. eduardii (QPE) }\end{array}$ & $\begin{array}{c}12.767> \\
\mathrm{t}_{(566)}=3.308\end{array}$ & $\begin{array}{c}9.361> \\
\mathrm{t}_{(620)}=3.306\end{array}$ & $\begin{array}{c}0.502< \\
t_{(607)}=3.306\end{array}$ \\
\hline
\end{tabular}

*Entre paréntesis se indican los grados de libertad a un nivel de significancia de 0.001 . Con negritas se señala la comparación en donde no hubo diferencias significativas. 


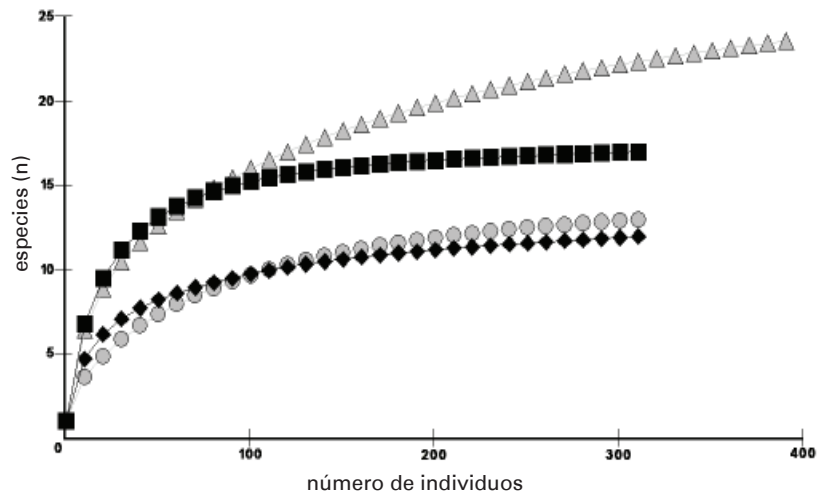

Figura 3. Curva de rarefacción para las asociaciones de encinares reconocidas en la sierra de Santa Rosa. Simbología: Quercus potosina-Q. castanea (triángulo), Q. potosina-Q. eduardii (cuadro), $Q$. coccolobifolia (círculo) y $Q$. laurina- $Q$. rugosa (rombo).

leñosos (arbóreo y arbustivo) formando 2 estratos más o menos bien definidos, aunque como bien lo señala Labat (1995) para los encinares michoacanos, la fisonomía de estas comunidades puede variar fuertemente de acuerdo con la etapa de regeneración que se observe. Asimismo, el patrón de dominancia de especies, con valores mayores al $70 \%$ en los índices de importancia, tanto para el estrato arbóreo como arbustivo, es similar al que otros autores registran en encinares (Rivas, 1980; Márquez et al., 1999; Loewenstein et al., 2000; García-Arévalo et al., 2004).

En cuanto a la densidad del estrato arbóreo, no existen tendencias entre los distintos bosques de encino, al menos para la región central de país. En el presente estudio se obtuvieron valores más altos (entre 622 y 1246 ind. ha $^{-1}$ ) que los obtenidos por Rivas (1980) en un estudio desarrollado para los encinares en Acámbaro, SE Guanajuato (159 a 681 ind. ha ${ }^{-1}$ ). Por el contrario, los valores de esta variable en la sierra de Santa Rosa son similares o menores a los que Labat (1995) determinó para los encinares del NO de Michoacán (900 a 2160 ind. ha-1). Diversos autores han postulado que las diferencias estructurales entre encinares, incluyendo la densidad de sus especies, se asocia a la influencia de factores climáticos y edáficos (Rzedowski, 1978; Angulo, 1985; Valiente-Banuet et al., 2000; Park, 2001; Encina y Villareal, 2002; Cotler, 2003), aunque es importante recordar el papel relevante de las actividades de aprovechamiento del hombre (McVaugh y Rzedowski, 1966; Rzedowski, 1978; Rubio, 1993; Labat, 1995; Escobar-Ocampo y Ochoa-Gaona, 2007). Por ejemplo, la alta densidad de arbustos (5 089 ind. ha ${ }^{-1}$ ) de la comunidad $Q$. potosina- $Q$. castanea puede estar generada por la producción de carbón in situ, ya que se ha postulado que esta situación favorece el crecimiento de
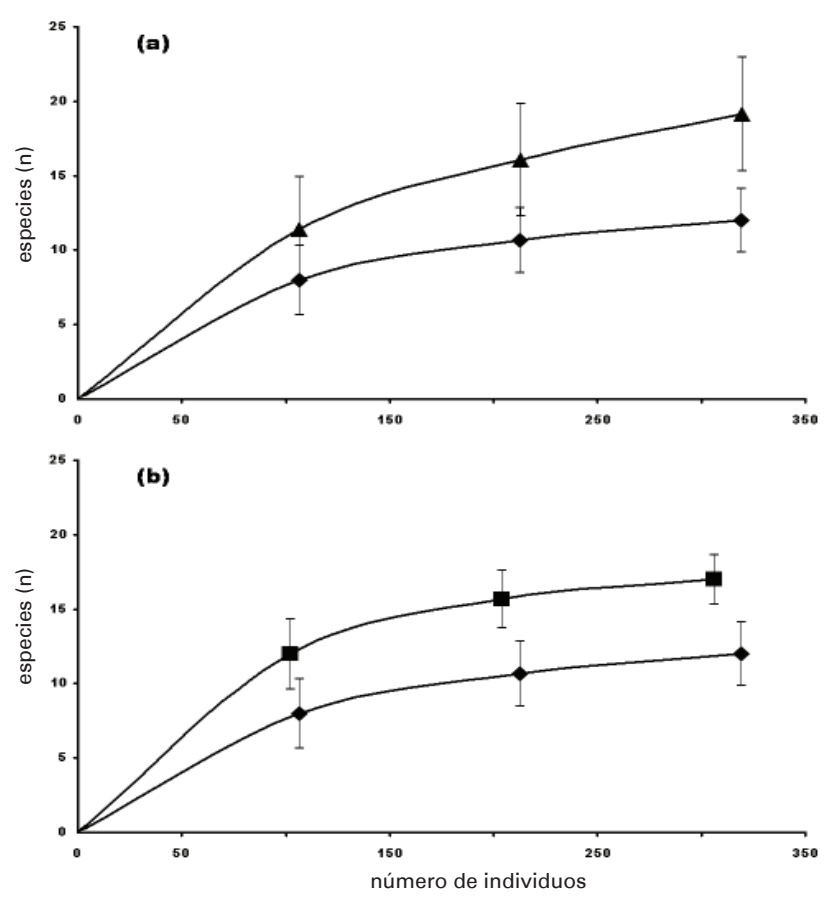

Figura 4. Asociaciones vegetales con diferencias en su diversidad florística, basados en un análisis de rarefacción de 50 iteraciones, con las barras de error de la varianza a un nivel de confianza de 95\%. Simbología: Quercus potosina-Q. castanea (triángulo), $Q$. potosina-Q. eduardii (cuadro), Q. laurina-Q. rugosa (rombo).

Cuadro 3. Valores de diversidad beta para las asociaciones reconocidas en los encinares en la sierra de Santa Rosa, utilizando el índice de Simpson (diagonal superior, en cursivas) y el índice de Sørensen cuantitativo (diagonal inferior)

\begin{tabular}{lcccc}
\hline Asociación & $Q C O$ & $Q L R$ & $Q P C$ & $Q P E$ \\
\hline $\begin{array}{l}\text { Quercus } \\
\text { coccolobifolia (QCO) }\end{array}$ & -- & 0.750 & 0.231 & 0.231 \\
$\begin{array}{l}\text { Quercus laurina-Q. } \\
\text { rugosa (QLR) }\end{array}$ & 0.031 & -- & 0.250 & 0.750 \\
$\begin{array}{l}\text { Quercus potosina-Q. } \\
\text { castanea (QPC) }\end{array}$ & 0.148 & 0.175 & -- & 0.118 \\
$\begin{array}{l}\text { Quercus potosina-Q. } \\
\text { eduardii (QPE) }\end{array}$ & 0.231 & 0.037 & 0.311 & -- \\
\hline
\end{tabular}

especies altamente tolerantes a suelos quemados, como Ageratina glabrata y Arctostaphylos pungens (Pineda, 1978; Márquez et al., 2006; Tarrega et al., 2006). Cambios drásticos en la composición y abundancia de especies vegetales características de los encinares, debido al uso intensivo por el hombre, han sido también documentados en localidades del continente europeo (Marañon et al., 
1999; Decocq et al. 2004).

Las diferentes medidas para estimar la diversidad de las asociaciones del área de estudio, tanto por número de especies, diversidad o equidad, coinciden en que las asociaciones de $Q$. potosina- $Q$. castanea y $Q$. potosina- $Q$. eduardii son las más sobresalientes (Cuadro 1, Figs. 3 y 4) y que la asociación de $Q$. coccolobioflia es una comunidad menos diversa; esto último también señalado por Quero (1977). Comparaciones directas del índice de Shannon muestran que los bosques del área de estudio obtuvieron valores más bajos (0.57-1.03) comparados con los obtenidos para encinares localizados al SE de Guanajuato (1.39-2.69), NO de Michoacán (2.56) y Colima (1.041.66) y en todos los casos, los valores mínimos obtenidos en estos estudios superan el máximo registrado para las asociaciones de la sierra de Santa Rosa (Rivas, 1980; Labat, 1995; Ramos, 2005). La equidad (E) obtenida en el estudio de Rivas (1980) fue de 0.61 a 0.84, en el de Labat (1995) de 0.59 y en los encinares de Colima (Ramos, 2005) entre 0.68 y 0.85 , todas ellas superiores a las registradas en el presente trabajo $(0.22-0.36)$.

En cuanto a la diversidad beta, independientemente del índice usado para evaluarla, las asociaciones reconocidas en el área estudiada mostraron un alto recambio de las especies que las constituyen (Cuadro 3), una situación compartida con los encinares del estado de Colima (Ramos, 2005). Lo anterior no concuerda con lo indicado por Halffter y Moreno (2005), quienes argumentan que numerosos grupos de organismos que habitan regiones templadas despliegan valores extremadamente bajos de diversidad beta. En resumen, las asociaciones que se delimitaron fisonómicamente en la sierra de Santa Rosa también difieren en otras variables estructurales (e. gr. densidad de individuos por hectárea o área basal promedio por individuo), aunado a sus diferencias en composición y abundancia de especies, que se reflejaron en los diferentes índices de diversidad calculados.

Los bosques de encino en Guanajuato abarcan una superficie de $9.3 \%$ (Palacio et al., 2000) y de ellos se obtiene una producción de carbón vegetal de $18885 \mathrm{~m}^{3}$, equivalente al $78.4 \%$ de toda la madera de encino aprovechada (Pérez et al., 2000). Sin embargo, estos autores subrayan que a pesar de la elevada producción, esta actividad es la de menor valor económico en el mercado laboral estatal. Esta disparidad incrementa el uso intensivo y extensivo de estos bosques, lo que puede llevar a un deterioro aún más severo del que se observa en este tipo de vegetación.

El presente trabajo está dirigido a promover estudios sobre la biodiversidad de los encinares mexicanos que faciliten comparaciones más amplias respecto a su composición, diversidad y estructura, asunto trascendental para México, debido a su importancia biológica (riqueza y endemismo), amplitud geográfica e interés económico. Se espera que la presente contribución colabore a la conformación de una propuesta de ordenamiento territorial que contemple un uso más sustentable de los encinares de la sierra de Santa Rosa.

\section{Agradecimientos}

A Ma. Guadalupe Cornejo Tenorio y 2 revisores anónimos, por las críticas a este trabajo que ayudaron a mejorar el contenido del manuscrito. Al M. en G. Jesús Fuentes Junco y al M. en C. Fernando Alvarado Ramos, por su asistencia en la elaboración de las figura 1. A la Comisión Nacional Forestal, por el acceso a su sistema de información geográfica para el estado de Guanajuato que permitió la elaboración de la figura 1 con la localización de la sierra de Santa Rosa. El primer autor desea externar su reconocimiento a la familia Cano Mares, por su hospitalidad y apoyo durante el trabajo de campo y a la Biól. Angélica Estrada Hernández, por su gestión para obtener la beca otorgada por el Consejo Internacional para la Conservación de las Aves en México (CIPAMEX) a fin de realizar este trabajo.

\section{Literatura citada}

Aguilera, L. I. 1991. Estudio florístico y sinecológico de la vegetación en el cráter Hoya de Rincón de Parangueo, Valle de Santiago, Guanajuato. Tesis Maestría Colegio de Postgraduados, Montecillo, Estado de México. 99 p.

Angulo, M. J. 1985. Relación clima-vegetación en el estado de Guanajuato. Tesis Facultad de Ciencias, UNAM, México, D. F. 106 p.

Carranza, E. 2005. Flora del Bajío y de Regiones Adyacentes. Fascículo complementario XXI. Conocimiento actual de la flora y la diversidad vegetal del estado de Guanajuato, México. Instituto de Ecología, Pátzcuaro, Michoacán. 17 p.

Colwell, R. K., C. Xuan y J. Chang. 2004. Interpolating, extrapolating and comparing incidence-based species accumulation curves. Ecology 85: 2717-2727.

Colwell, R. K. 2005. EstimateS: statistical estimation of species richness and shared species from samples. Version 7.5. User's guide and application: http://purl.oclc.org/estimates Consultado. 03 julio 2007.

Cotler, H. 2003. Características y manejo de suelos en ecosistemas templados de montaña. In Conservación de ecosistemas templados de montaña en México, O. Sánchez, E. Vega, E. Peters y O. Monroy-Vilchis (eds.) Instituto Nacional de Ecología, México, D. F. p. 153-161.

Cuanalo de la Cerda, H., E. Ojeda, A. Santos y C. A. Ortiz. 1989. Provincias, regiones y subregiones terrestres de México. Colegio de Posgraduados, Montecillo, Estado de México. 
$624 \mathrm{p}$.

Danserau, P. 1957. Biogeography and ecological perspective. Ronald, New York. 394 p.

Dávila, P. y V. Sosa. 1994. El conocimiento florístico de México. Boletín de la Sociedad Botánica de México 55:1-28.

Decocq, G., M. Aubert, F. Dupont, D. Alard, R. Saguez, A. Wattez-Franger, B. de Foucault, A. Delelis-Dusollier y J. Bardat. 2004. Plant diversity in a managed temperate deciduous forest: understory response to two silvicultural systems. Journal of Applied Ecology 41:1065-1079.

Encina, J. A. y J. A. Villareal. 2002. Distribución y aspectos ecológicos del género Quercus (Fagaceae) en el estado de Coahuila. Polibotánica 13:1-23.

Enríquez E. D. 2003. Flora y vegetación de la sierra de Órganos, municipio de Sombrerete, Zacatecas, México. Acta Botanica Mexicana 64: 45-89.

Escobar-Ocampo, Ma. Consuelo y S. Ochoa-Gaona. 2007. Estructura y composición florística de la vegetación del Parque Educativo Laguna Bélgica, Chiapas, México. Revista Mexicana de Biodiversidad 78: 391-419.

Ferrusquía-Villafranca, I. 1993. Geology of Mexico: a sinopsis. In Biological diversity of Mexico: origins and distribution, T. P. Ramamoorthy, R. Bye, A. Lot y J. Fa (eds.) Oxford University Press, New York. p. 3-107.

García-Arévalo, A., J. J. Mendoza-Contreras y J. Nocedal. 2004. Asociaciones vegetales del municipio de Guanacevi, Durango. Madera y Bosques 10:21-34.

García, E. 1981. Modificaciones al sistema de clasificación climática de Köppen, Tercera edición. Instituto de Geografía, UNAM, México, D. F. 252 p.

Halffter, G. y C. E. Moreno. 2005. Significado biológico de las diversidades alfa, beta y gamma. In Sobre diversidad biológica: el significado de las diversidades alfa, beta y gamma. G. Halffter, J. Soberón, P. Koleff y A. Melic (eds.) m3m-Monografías tercer milenio, vol. 4, Sociedad Entomológica Aragonesa, Zaragoza/Comisión Nacional para el Conocimiento y Uso de la Biodiversidad/Grupo Diversitas-México/CONACYT, México, D. F. p. 5-18.

INEGI (Instituto Nacional de Estadística, Geografía e Informática). 1991. Datos básicos de la Geografía de México. Instituto Nacional de Estadística Geografía e Informática, Aguascalientes, Aguascalientes. $142 \mathrm{p}$.

Kappelle, M. 1996. Los bosques de roble (Quercus) de la cordillera de Talamanca, Costa Rica. Biodiversidad, ecología, conservación y desarrollo. Universidad de Ámsterdam e Instituto Nacional de Biodiversidad, Wageningen. $319 \mathrm{p}$.

Koleff, P., K. J. Gaston y J. J. Lennon. 2003. Measuring beta diversity for presence-absence data. Journal of Animal Ecology 72:367-382.

Koleff, P. 2005. Conceptos y medidas de la diversidad beta. In Sobre diversidad biológica: el significado de las diversidades alfa, beta y gamma. G. Halffter, J. Soberón, P. Koleff y A. Melic (eds.). m3m-Monografías Tercer milenio, volumen 4, Sociedad Entomológica Aragonesa, Zaragoza/Comisión Nacional para el Conocimiento y Uso de la Biodiversidad/Grupo DiversitasMéxico/ CONACYT, México, D. F. p. 19-40.

Labat, J. N. 1995. Flora del Bajío y de Regiones Adyacentes.
Fascículo complementario VIII. Végétation du nord-ouest du Michoacán Mexique. Instituto de Ecología, Pátzcuaro, Michoacán. 401 p.

Loewenstein, E. F.; P. S. Jonson y H. E. Garret. 2000. Age and diameter structure of managed uneven-agen oak forest. Canadian Journal Forest Research 30:1060-1070.

Magurran, A. E. 2004. Measuring biological diversity. Blackwell, Oxford. $256 \mathrm{p}$.

Marañon, T., R. Ajbilou, F. Ojeda y J. Arroyo. 1999. Biodiversity of woody species in oak woodlands of southern Spain and northern Morocco. Forest Ecology and Management 115:147-156.

Márquez, M. A., S. González y R. Álvarez. 1999. Componentes de la diversidad arbórea en bosques de pino-encino de Durango, México. Madera y Bosques 5:67-78.

Márquez, M. A., E. Jurado y S. González. 2006. Algunos aspectos de la biología de la manzanita (Arctostaphylos pungens HBK.) y su papel en el desplazamiento de bosques templados por chaparrales. Ciencia 9:57-64.

Martínez-Cruz, J. y O. Téllez. 2004. Listado florístico de la sierra de Santa Rosa, Guanajuato, México. Boletín de la Sociedad Botánica de México. 74:31-49.

McAleece, N. 1997. Biodiversity professional beta. Natural History Museum and Scottish Association for Marine Science, Oban, Argyll.

McVaugh, R. y J. Rzedowski. 1966. La vegetación de Nueva Galicia. Contributions of University of Michigan Herbarium 9:1-123.

Moreno, C. E. 2001. Manual de métodos para medir la biodiversidad. Manuales y Tesis Sociedad Entomológica Aragonesa, Zaragoza. 84 p.

Mueller-Dombois, D. y H. Ellenberg. 1974. Aims and methods of vegetation ecology. John Wiley, New York. 541 p.

Palacio-Prieto, J. L., G. Bocco, A. Velásquez, J. F. Mass, F. Takaki-Takaki, A. Victoria, L. Luna-González, G. GómezRodríguez, J. López-García, M. Palma, I. Trejo-Vázquez, A. Peralta, J. Prado-Molina, A. Rodríguez-Aguilar, R. MayorgaSaucedo y F. González-Medrano. 2000. La condición actual de los recursos forestales en México: resultados del Inventario Forestal Nacional 2000. Investigaciones Geográficas, Boletín del Instituto de Geografía Universidad Nacional Autónoma de México 43:183-203.

Park, A. D. 2001. Environmental influences on post-harvest natural regeneration in Mexican pine-oak forest. Forest Ecology and Management 144:213-228.

Pérez, O. M., R. Dávalos y E. Guerrero. 2000. Aprovechamiento de la madera en México. Madera y Bosques 1:6-13.

Pineda, R. A. 1978. La vegetación forestal del estado de Guanajuato. Bosques y Fauna 1:31-41.

Quero, R. J. 1977. La vegetación de las serranías de la Cuenca Alta del Río de la Laja, Guanajuato. Anales del Instituto de Biología, Universidad Nacional Autónoma de México, Serie Botánica 47-53:73-99.

Ramos, L. A. 2005. Diversidad y conservación de los encinares de Colima, México. Tesis Maestría Posgrado en Ciencias Biológicas, Universidad Nacional Autónoma de México, México. $61 \mathrm{p}$.

Rivas, H. A. 1980. Estudio sinecológico del municipio de 
Acámbaro, Guanajuato. Tesis Escuela de Biología, Universidad Michoacana de San Nicolás de Hidalgo, Morelia, Michoacán. 85 p.

Rubio, C. A. 1993. Contribución al estudio florístico de la sierra de los Agustinos, Guanajuato, México. Tesis Escuela de Biología, Universidad Michoacana de San Nicolás de Hidalgo, Michoacán. 87 p.

Rzedowski, J. 1978. Vegetación de México. Limusa, México, D. F. 432 p.

Rzedowski, J. 1991. Diversidad y orígenes de la flora fanerogámica de México. Acta Botanica Mexicana 14:3-21.

Rzedowski, J., G. Calderón de Rzedowski y R. Galván. 1995. Flora del Bajío y de Regiones Adyacentes. Fascículo complementario IX. Los pastizales calcífilos del estado de Guanajuato. Instituto de Ecología, Pátzcuaro, Michoacán. 19 p.

Rzedowski, J., G. Calderón de Rzedowski y R. Galván 1996. Flora del Bajío y de Regiones Adyacentes. Fascículo complementario XIV. Nota sobre la vegetación y la flora del noreste del estado de Guanajuato. Instituto de Ecología, Pátzcuaro, Michoacán. 22 p.

SPP (Secretaría de Programación y Presupuesto). 1981. Síntesis geográfica del estado de Guanajuato. Coordinación General de Estadística, Geografía e Informática, México, D.F. 198 p.

Tarrega R., L. Calvo, E. Marcos y A. Tabeada. 2006. Forest structure and understory diversity in Quercus pyrenaica communities with different human uses and disturbances. Forest Ecology and Management 227:50-58.

Valiente-Banuet, A., A. Casas, A. Alcántara, P. Dávila, N. Flores, M. Coro, J. L. Villaseñor y J. Ortega. 2000. La vegetación del valle de Tehuacán-Cuicatlán. Boletín de la Sociedad Botánica de México 67:25-74.

Villaseñor, J. L. 2003. Diversidad y distribución de las Magnoliophyta de México. Interciencia 28:160-167.

Apéndice 1. Árboles y arbustos en 4 asociaciones de encinos en la sierra de Santa Rosa, Guanajuato. Valores estructurales absolutos para frecuencia $(F r)$, densidad $(D e)$ y dominancia ( $D o$, área basal para árboles y cobertura para arbustos). Índice de importancia $(I D I)$. Especies exclusivas de una asociación, con asterisco (*).

\begin{tabular}{llccc}
\hline Especies & $\begin{array}{c}F r \\
(\%)\end{array}$ & $\begin{array}{c}\text { De } \\
\left(\text { Ind. } h a^{-1}\right)\end{array}$ & $\begin{array}{c}\text { Do } \\
\left(m^{2}\right)\end{array}$ & $\begin{array}{c}I D I \\
(\%)\end{array}$ \\
\hline
\end{tabular}

Asociación Quercus coccolobifolia

Árboles

Quercus coccolobifolia Trel.

$\begin{array}{cccc}100 & 1067 & 0.891 & 77.3 \\ 23 & 86 & 0.085 & 9.8 \\ 10 & 39 & 0.092 & 6.0 \\ 8 & 23 & 0.029 & 3.2 \\ 8 & 23 & 0.018 & 2.8 \\ 3 & 8 & 0.004 & 0.9\end{array}$

Arbutus glandulosa M. Martens et Galeotti

8

Quercus eduardii Trel.

\section{Arbustos}

Arctostaphylos pungens Kunth

Quercus coccolobifolia Trel.

40.175

Comarostaphylis polifolia (Kunth) Zucc. ex Klotzsch *

Pinus cembroides Zucc.

Rhamnus microphylla Humb. et Bonpl. ex Schult.

Stevia lucida Lag.

Comarostaphylis glaucescens (Kunth) Zucc. Ex Klotzsch

Asociación Quercus laurina-Quercus rugosa

\section{Árboles}

Quercus laurina Humb. et Bonpl.

$\begin{array}{cccc}88 & 567 & 3.304 & 55.4 \\ 83 & 531 & 1.404 & 40.5 \\ 8 & 28 & 0.039 & 2.5 \\ 3 & 7 & 0.134 & 1.6\end{array}$

Garrya laurifolia Hartw. ex Benth. 
Apéndice 1. Continúa

\begin{tabular}{lcccc}
\hline Especies & $\begin{array}{c}F r \\
(\%)\end{array}$ & $\begin{array}{c}\text { De } \\
\left(\text { Ind. } h a^{-1}\right)\end{array}$ & $\begin{array}{c}\text { o } \\
\left(\mathrm{m}^{2}\right)\end{array}$ & $\begin{array}{c}\text { IDI } \\
(\%)\end{array}$ \\
\hline
\end{tabular}

Asociación Quercus laurina-Quercus rugosa

\section{Arbustos}

Ageratina sp. $1 *$

$\begin{array}{lccc}28 & 874 & 34.510 & 26.2 \\ 65 & 1853 & 2.619 & 21.1 \\ 48 & 874 & 10.509 & 16.9 \\ 23 & 594 & 9.959 & 11.5 \\ 35 & 629 & 2.008 & 9.5 \\ 23 & 315 & 3.599 & 6.7 \\ 10 & 175 & 1.090 & 2.9 \\ 8 & 140 & 1.228 & 2.5 \\ 5 & 70 & 0.073 & 1.1 \\ 3 & 35 & 1.080 & 1.1 \\ 3 & 35 & 0.015 & 0.6\end{array}$

Quercus laurina Bonpl.

Roldana angulifolia (DC.) H. Rob. et Brettell *

Stevia lucida

Cestrum anagyris Dunal

Quercus rugosa Née

Buddleja cordata Kunth

Ageratina glabrata

Arbutus glandulosa

Ilex dugesii Fernald *

Cornus excelsa Kunth

Asociación Quercus potosina-Quercus castanea

\section{Árboles}

Quercus potosina

90

Quercus castanea Née

Quercus laurina

Arbutus glandulosa

Quercus rugosa

Quercus sideroxyla

Quercus coccolobifolia

Prunus serotina Ehrh. *

Crataegus mexicana Moc. et Sessé ex DC. *

Quercus laeta Liebm. *

Alnus acuminata Kunth *

Arbutus xalapensis

\section{Arbustos}

Ageratina glabrata

Baccharis heterophylla Kunth

Arctostaphylos pungens

Stevia salicifolia Cav.

Quercus potosina

Arbutus glandulosa

Amelanchier denticulada (Kunth) K. Koch

Quercus laurina

Stevia lucida

Quercus castanea

Rhamnus microphylla

Ageratina sp. 2

\section{9}

28

30

11

5

4

3

4

1

1

1

72

42

26

22

18

19

8

10

8

6

7

4

299
166
75
57
19
8
10
5
6
3
1
1

5.654

43.1

3.541

26.3

1.697

12.3

0.693

9.0

0.242

3.1

0.140

1.5

0.096

1.4

0.206

1.3

0.089

0.084

0.059

1.1

0.6

0.003

0.3

0.1

311.038

35.6

779

104.834

15.3

588

122.770

12.8

413

24.322

6.6

239

38.147

5.6

254

29.847

5.4

143

16.250

2.6

127

8.920

2.5

135

6.555

2.5
2.2

95

9.608

2.2

111

3.633

1.9

72

11.020 
Apéndice 1. Continúa

\begin{tabular}{|c|c|c|c|c|}
\hline Especies & $\begin{array}{l}F r \\
(\%)\end{array}$ & $\begin{array}{c}D e \\
\left(\text { Ind. } h a^{-1}\right)\end{array}$ & $\begin{array}{l}D o \\
\left(m^{2}\right)\end{array}$ & $\begin{array}{l}I D I \\
(\%)\end{array}$ \\
\hline \multicolumn{5}{|c|}{ Asociación Quercus potosina-Quercus castanea } \\
\hline Cornus excelsa & 3 & 48 & 12.853 & 1.3 \\
\hline Havardia elachystophylla A. Gray & 6 & 80 & 0.893 & 1.3 \\
\hline Crataegus mexicana & 3 & 32 & 1.815 & 0.6 \\
\hline Comarostaphylis glaucescens & 1 & 16 & 5.080 & 0.5 \\
\hline Dodonaea viscosa Jacq. * & 2 & 24 & 1.700 & 0.5 \\
\hline Cestrum anagyris & 2 & 24 & 1.080 & 0.4 \\
\hline Buddleja cordata & 1 & 16 & 1.323 & 0.3 \\
\hline Salvia mexicana L. * & 1 & 16 & 0.900 & 0.3 \\
\hline Quercus coccolobifolia & 1 & 16 & 0.143 & 0.3 \\
\hline Arbutus xalapensis & 1 & 8 & 2.530 & 0.2 \\
\hline Ageratina petiolaris (Moc. et Sessé ex DC.) R.M. King et H. Rob. * & 1 & 8 & 1.320 & 0.2 \\
\hline Garrya laurifolia & 1 & 8 & 0.020 & 0.1 \\
\hline \multicolumn{5}{|c|}{ Asociación Quercus potosina-Quercus eduardii } \\
\hline \multicolumn{5}{|c|}{ 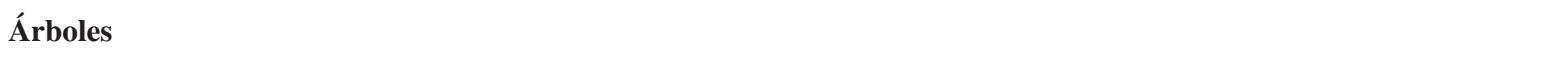 } \\
\hline Quercus potosina & 98 & 324 & 1.667 & 47.9 \\
\hline Quercus eduardii & 70 & 189 & 0.834 & 28.3 \\
\hline Quercus coccolobifolia & 30 & 74 & 0.138 & 9.5 \\
\hline Quercus castanea & 15 & 29 & 0.371 & 7.5 \\
\hline Arbutus glandulosa & 13 & 25 & 0.095 & 4.0 \\
\hline Quercus sideroxyla & 8 & 16 & 0.085 & 2.8 \\
\hline \multicolumn{5}{|l|}{ Arbustos } \\
\hline Arctostaphylos pungens & 38 & 664 & 64.180 & 25.6 \\
\hline Ageratina glabrata & 53 & 771 & 44.567 & 24.3 \\
\hline Stevia salicifolia & 35 & 492 & 10.564 & 11.7 \\
\hline Baccharis heterophylla & 23 & 278 & 12.100 & 8.4 \\
\hline Ageratina calaminthaefolia (Kunth) R.M. King et H. Rob. * & 25 & 321 & 5.793 & 7.7 \\
\hline Rhamnus microphylla & 25 & 300 & 1.836 & 6.6 \\
\hline Havardia elachystophylla & 15 & 150 & 0.228 & 3.5 \\
\hline Ageratina sp. 2 & 10 & 150 & 2.514 & 3.3 \\
\hline Quercus eduardii & 8 & 86 & 4.590 & 2.8 \\
\hline Stevia lucida & 10 & 107 & 1.863 & 2.8 \\
\hline Amelanchier denticulada & 5 & 43 & 0.895 & 1.3 \\
\hline Arbutus xalapensis & 3 & 21 & 0.990 & 0.8 \\
\hline Arbutus glandulosa & 3 & 21 & 0.350 & 0.6 \\
\hline Quercus castanea & 3 & 21 & 0.240 & 0.6 \\
\hline
\end{tabular}

